\title{
Cytomegalovirus retinitis in chronic lymphocytic leukaemia
}

\begin{abstract}
Purpose To describe a rare association of cytomegalovirus (CMV) retinitis. Patients and methods Report of three patients with chronic lymphocytic leukaemia (CLL) who developed CMV retinitis.

Results The diagnosis was established by the detection of CMV DNA by polymerase chain reaction analysis of aqueous and/or vitreous humour. CD4 + T-lymphocyte count was reduced in two patients and normal in the third one. There was bilateral involvement in two of the three patients. There was delay of 8-10 months in diagnosis. The visual outcome was poor in four out of the five eyes involved owing to optic atrophy or total retinal detachment with proliferative vitreoretinopathy. Conclusion Increased awareness of CMV retinitis in patients who have received immunosuppression for haematological malignancies, such as CLL, could lead to earlier diagnosis and possibly better visual outcome.
\end{abstract}

Eye (2007) 21, 1230-1233; doi:10.1038/sj.eye.6702550;

Birmingham and Midland Eye Centre, Sandwell and West Birmingham NHS

Trusts, Birmingham, UK

Correspondence: P Stavrou, Birmingham and Midland Eye Centre,

Sandwell and West Birmingham NHS Trusts, Dudley Road, Birmingham B18 7QH, UK Tel: + 44121 5076854; Fax: + 441215076853 E-mail: panagiotastavrou@ hotmail.com

Received: 31 January 2006 Accepted in revised form: 10 July 2006

Published online: 18 August 2006 published online 18 August 2006

Keywords: cytomegalovirus retinitis; chronic lymphocytic leukaemia; polymerase chain reaction; ganciclorir; T-lymphocyte count; immunodeficiency

\section{Introduction}

Cytomegalovirus (CMV) retinitis is a sightthreatening disease known to occur in patients with immunodeficiency. ${ }^{1-4}$ We report three cases of severe visual loss owing to CMV retinitis in patients with chronic lymphocytic leukaemia (CLL).
J Church, S Goyal, AK Tyagi, RAH Scott and P Stavrou

Patients and methods

Case 1

A 76-year-old Caucasian man noticed floaters during 3-month hospitalization for newly diagnosed CLL. The treatment of the haematological malignancy had been systemic steroids, intravenous immunoglobulins, and monoclonal anti-CD20 antibody. After discharge from the haematological ward, he was assessed by the local ophthalmologists who diagnosed bilateral panuveitis and secondary glaucoma and treated him with a 4-month course of oral steroids and topical hypotensives. Owing to poor response to oral steroids, he underwent right diagnostic vitrectomy and lensectomy. Cytopathologic analysis of vitreous aspirate and choroidal biopsy did not show evidence of malignancy. At this stage, no sample was sent for polymerase chain reaction (PCR) analysis to exclude intraocular infection. Eight months later, owing to ongoing inflammation he was referred to us.

Visual acuity was right eye: 2/60 and left eye: $1 / 60$. There was bilateral dense vitritis, disc pallor, and areas of retinitis associated with retinal haemorrhages (Figure 1a and b). Intraocular pressure was controlled on two types of ocular hypotensives. PCR analysis of vitreous aspirate was positive for CMV DNA (1.6 million DNA copies $/ \mathrm{ml}$ ). Aqueous humour analysis by PCR was also positive for CMV DNA (40000 DNA copies/ml). PCR analysis of peripheral blood was negative for CMV DNA. Further analysis of blood samples disclosed normal absolute lymphocyte count: $1.03 \times 10^{9} / 1$; reduced CD $4+$ count: $0.35 \times 10^{9} / 1$ (laboratory range: $\left.0.40-1.80 \times 10^{9} / 1\right)$; and normal $C D 4+/$ CD8 + ratio of 1.67 (reference range: 0.66-3.52). The patient was initiated on intravenous ganciclovir ( $5 \mathrm{mg} / \mathrm{kg}$ every $12 \mathrm{~h}$ ) for 2 weeks, which was followed by oral valganciclovir (900 mg once a day) for another 3 months. He responded to the treatment, the vitritis settled 


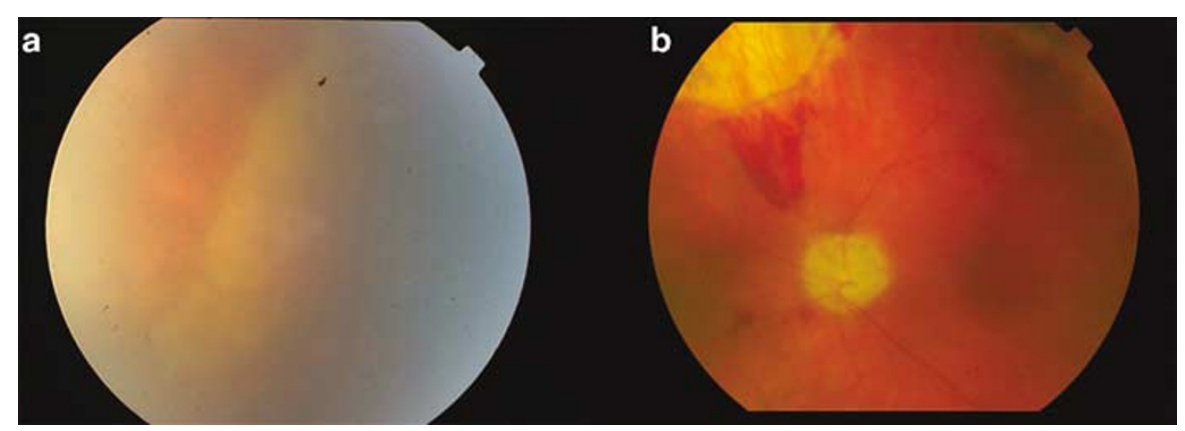

Figure 1 (a) Case 1. Nasal periphery of right fundus showing active retinitis. (b) Case 1. Posterior pole of left eye showing retinal haemorrhages, an area of healed retinitis, and optic atrophy.

down, and the retinitis arrested leaving extensive areas of chorioretinal atrophy. At 26 months of follow-up, visual acuity was right eye $3 / 60$ and left eye $1 / 60$ due to bilateral optic atrophy. This was thought to be due to combination of $360^{\circ}$ retinitis, which extended centrally and raised intraocular pressure.

\section{Case 2}

A 67-year-old Caucasian female presented with floaters in the right eye. She was known to suffer from CLL for 2.5 years and had been treated with two courses of chemotherapy (chlorambucil, fludarabine, and prednisolone). The first course of chemotherapy was given at diagnosis of CLL and the second one was completed a month before her developing ocular symptoms. A clinical diagnosis of viral retinitis was made by the local ophthalmologist who initiated empiric treatment with oral aciclovir (400 mg twice a day), increased the dose of steroids (prednisolone $60 \mathrm{mg}$ a day), and referred the patient to us.

Visual acuity was right eye: $6 / 6$ and left eye: $6 / 5$. In the right eye, there was an area of retinitis in the temporal periphery (Figure 2). Examination of the left eye was unremarkable. An aqueous sample was negative by PCR for viruses, candida, and cocci. As the area of retinitis appeared to be healing, the aciclovir was continued for another 4 months.

Three months later, the patient suffered reduction of right visual acuity associated with increase of vitreous cellular activity and areas of retinitis in the periphery. A second aqueous sample was positive for CMV DNA (250000 DNA copies/ml) by PCR. PCR analysis of peripheral blood was negative for CMV. Further analysis of blood samples showed normal absolute lymphocyte count: $1.58 \times 10^{9} / 1$, reduced CD4 + count: $0.33 \times 10^{9} / 1$ (laboratory range: $0.40-1.80 \times 10^{9} / 1$ ), and normal CD4 + /CD8 + ratio of 1.17 (reference range: 0.66-3.52).

The patient was initiated on ganciclovir, which was given intravenously $(5 \mathrm{mg} / \mathrm{kg}$ every $12 \mathrm{~h}$ ) for the first 2 weeks followed by oral valganciclovir ( $900 \mathrm{mg}$ once



Figure 2 Case 2. Temporal periphery of right fundus showing active retinitis.

a day). Repeat blood monitoring showed progressive bone marrow suppression, and at 6 weeks, valganciclovir was stopped due to pancytopaenia: white cell count: $2 \times 10^{9}$ / l; neutrophils absolute count: $0.44 \times 10^{9} / 1$; lymphocytes absolute count: $1.28 \times 10^{9} / \mathrm{l} ; \mathrm{Hb}: 11.3 \mathrm{~g} / \mathrm{dl}$; and platelets: $199 \times 10^{9} / 1$. Following bone marrow biopsy, the patient was treated with several cycles of intravenous immunoglobulins, intravenous vincristine, and granulocyte colony-stimulating factor ( $\times 3$ per week). By that time, the patient had developed a white cataract, which made intravitreous injections of anti-CMV agents impossible. Subsequently, the patient underwent implantation of a ganciclovir implant combined with phacoemulsification and intraocular lens implantation. A preoperative platelet transfusion was used to control bleeding. Despite arrest of retinitis, visual acuity at 24 months of follow-up remains hand movements due to optic atrophy.

\section{Case 3}

An 85-year-old-Caucasian man with CLL for 3 years was referred to us with significant vitritis following routine right phacoemulsification. The CLL had been managed with oral cyclophosphamide and intravenous 
immunoglobulins. Visual acuity was right eye: 1/60 and left eye: 6/12. There was dense vitritis and tractional retinal detachment along superotemporal vascular arcade in the right eye, while the left eye was quiet. CMV DNA (900000 DNA copies/ml) was identified by PCR analysis of a vitreous specimen. No peripheral blood specimen was sent for PCR examination. Analysis of blood samples showed high absolute lymphocyte count: $38.28 \times 10^{9} / 1$ (laboratory range: $0.40-1.80 \times 10^{9} / 1$ ); normal CD4 + count: $0.88 \times 10^{9} / 1$ (laboratory range: $\left.0.40-1.80 \times 10^{9} / 1\right)$; and normal CD4 + /CD8 + ratio of two (reference range: $0.66-3.52$ ).

The patient was initiated on valganciclovir $(900 \mathrm{mg}$ twice a day for 2 weeks followed by $900 \mathrm{mg}$ once a day). However, 2 months later, visual acuity in the right eye deteriorated to no perception of light owing to rubeosis iridis, total retinal detachment, and proliferative vitreoretinopathy. The valganciclovir was discontinued when the patient's general health deteriorated and required hospitalization elsewhere with severe pneumonia, which was considered bacterial on the basis of cultures from sputum.

A month later, the patient represented with floaters in his second (left) eye. Examination revealed a new area of retinitis in the superior periphery. CMV DNA (1700 CMV DNA copies $/ \mathrm{ml}$ ) was identified by PCR analysis of aqueous sample. Simultaneous analysis of peripheral blood demonstrated 11500 CMV DNA copies/ml. The patient was re-started on oral valganciclovir $(900 \mathrm{mg}$ once a daily), while oral cyclophosphamide was discontinued. Valganciclovir was discontinued 4 months later when the area of retinitis appeared inactive, the vitreous had cleared, and visual acuity was 6/24.

\section{Discussion}

Patients with CLL may be immunocompromised because of the disease itself or its treatment. Triple viral retinitis identified by PCR of vitreous biopsy was reported in a patient with CLL who had undergone malignant lymphomatous transformation. ${ }^{5}$ Leukaemic infiltration of the eye can also occur in leukaemias and may be difficult to differentiate from infectious retinitis.

Classically, CMV retinitis, especially in patients with AIDS, is characterized by minimal or no cellular activity in the vitreous or anterior chamber. However, moderate vitritis was reported in two patients with CMV retinitis associated with Good syndrome, ${ }^{6}$ and vitritis, periphlebitis, retinitis, and serous macular detachment were noted in a patient with CMV retinitis and Hodgkin's disease. ${ }^{7}$ A different immunological profile and the late presentation of our patients may explain the marked vitritis noted in all of them.
The CD4 + T-lymphocyte count was reduced in two patients and normal in the third one in this series. Depression of CD4 + T-lymphocyte and CD8+ T-lymphocyte counts, in the range of $0.15-0.2 \times 10^{9} / 1$, is well documented in patients with CLL after chemotherapy, especially fludarabine. ${ }^{8}$ In the same study, it was reported that although recovery of CD4 + and CD8 + counts towards normal levels is slow, the incidence of all infections is low in patients in remission (one episode of infection for every 3.33 patient years at risk) and decreases with time off treatment. ${ }^{8}$ Furthermore, there was no association of infections or febrile episodes with the CD4 + count at the end of treatment and a poor correlation with the increase in CD4 + counts during remission. ${ }^{8}$ Thus, the risk of infection appears to be low during remission, but vigilance for opportunistic infections is always necessary.

It has been reported that in patients on immunosuppressive treatment developing CMV retinitis reduction of dose or discontinuation of immunosuppressive treatment, without specific antiCMV therapy, may be sufficient to arrest the retinitis. ${ }^{5}$ In Case 3, discontinuation of cyclophosphamide may have helped to control the CMV retinitis in the second eye. Another important issue in treating CMV retinitis in patients with CLL is that of valganciclovir-induced myelosuppression. Case 2 developed pancytopaenia, which required discontinuation of valganciclovir and granulocyte colony-stimulating factor. These results emphasize the need for close collaboration between ophthalmologists and haematologists so that myelosuppresion can be identified early and treated.

As indications for immunosuppression and organ transplant increase, the incidence of CMV retinitis in non-HIV-immunosuppressed patients is expected to rise. Increased awareness of CMV retinitis among those dealing with patients with haematological malignancies, such as CLL, may result in earlier diagnosis and improve visual outcome.

\section{References}

1 Ng P, McCluskey P, McCaughan G, Granville A, MacDonald $\mathrm{P}$, Keogh A. Ocular complications of heart, lung and liver transplantation. Br J Ophthalmol 1998; 82: 423-428.

2 Okamoto T, Okada M, Mori A, Saheki K, Takatsuka H, Wada $\mathrm{H}$ et al. Successful treatment of severe cytomegalovirus retinitis with foscarnet and intraocular injection of ganciclovir in a myelosuppressed unrelated bone marrow transplant patient. Bone Marrow Transplant 1997; 20: 801-803.

3 Kuo IC, Kempen JH, Dunn JP, Vogelsang G, Jabs DA. Clinical characteristics and outcomes of cytomegalovirus retinitis in persons without Human Immunodeficiency Virus infection. Am J Ophthalmol 2004; 138: 338-346. 
4 Ganly PS, Arthur C, Goldman JM, Schulenburg WE. Foscarnet as treatment for cytomegalovirus retinitis following bone marrow transplantation. Postgrad Med J 1988; 64: 389-391.

5 Levinson RD, Hooks JJ, Wang Y, Chiu MT, Kellaway J, Chan CC. Triple viral retinitis diagnosed by polymerase chain reaction of the vitreous biopsy in a patient with Richter syndrome. Am J Ophthalmol 1998; 126: 732-733.
6 Assi AC, Lightman S. Cytomegalovirus retinitis in patients with Good syndrome. Arch Ophthalmol 2002; 120: 510-512.

7 Nasir MA, Jaffe GJ. Cytomegalovirus retinitis associated with Hodgkin's disease. Retina 1996; 16: 324-327.

8 Keating MJ, O'Brien S, Lerner S, Koller C, Beran M, Robertson LE et al. Long-term follow-up of patients with chronic lymphocytic leukemia (CLL) receiving fludarabine regimens as initial therapy. Blood 1998; 92: 1165-1171. 